\title{
Integrative analysis of mRNA and miRNA expression profiles reveals seven potential diagnostic biomarkers for non-small cell lung cancer
}

\author{
JIE ZHANG $^{1 *}$, DONG LI ${ }^{2 *}$, YUEMING ZHANG ${ }^{1}$, ZONGLI DING ${ }^{1}$, \\ YULONG ZHENG $^{1}$, SONG CHEN ${ }^{3}$ and YUFENG WAN ${ }^{1}$ \\ Departments of ${ }^{1}$ Respiratory Diseases, ${ }^{2}$ Ear-Nose-Throat, The Affiliated Huai'an Hospital of Xuzhou Medical University, \\ Huai'an, Jiangsu 223002; ${ }^{3}$ Institute of Medicinal Biotechnology, Jiangsu College of Nursing, \\ Huai'an, Jiangsu 223300, P.R. China
}

Received April 25, 2019; Accepted October 7, 2019

DOI: 10.3892/or.2019.7407

\begin{abstract}
The present study aimed to explore specific molecular targets for the diagnosis and treatment of non-small cell lung cancer (NSCLC). The expression profiles of microRNAs (miRNAs) and mRNAs were downloaded from the GEO (GSE102286 and GSE101929) and TCGA databases. After data preprocessing, differentially expressed genes (DEGs) and differentially expressed miRNAs (DEMs) in cancer and normal tissues were selected and used to construct a DEM-DEG regulatory network and a protein-protein interaction (PPI) network. The genes and miRNAs in these networks were subjected to functional enrichment and survival analyses. Several key DEMs and DEGs were verified using RT-qPCR, and the results were statistically interpreted using a multivariate logistic regression analysis. In this study, 25 DEMs and 789 DEGs common to all datasets were identified, which were then used for the construction of a DEM-DEG regulatory network and a PPI network module. Survival analyses of 19 DEMs in the DEM-DEG regulatory network and 36 DEGs in the PPI network module revealed that 34 DEGs (including TOP2A, CCNB1, BIRC5, and TTK) and two miRNAs (miR-21-5p and miR-31-5p) were significantly associated with NSCLC prognosis. Moreover, RT-qPCR analysis identified three DEGs and five DEMs that had changes in
\end{abstract}

Correspondence to: Professor Yufeng Wan, Department of Respiratory Diseases, The Affiliated Huai'an Hospital of Xuzhou Medical University, Huai'an, Jiangsu 223002, P.R. China

E-mail: ggwanyufeng@163.com

Professor Song Chen, Institute of Medicinal Biotechnology, Jiangsu College of Nursing, Huai'an, Jiangsu 223300, P.R. China

E-mail: biocs@163.com

${ }^{*}$ Contributed equally

Key words: non-small cell lung cancer, mRNA, microRNA, network, multivariate logistic regression analysis expression consistent with those observed in the bioinformatic analysis. Finally, a multivariate logistic regression analysis of the data showed that TOP2A, CCNB1, BIRC5, miR-21-5p, miR-193b-3p, miR-210-3p and miR-31-5p could be combined for the diagnosis of NSCLC. In conclusion, TOP2A, CCNB1, BIRC5, miR-21-5p, miR-193b-3p, miR-210-3p and miR-31-5p may therefore serve as important biomarkers and diagnostic targets for NSCLC.

\section{Introduction}

Lung cancer is the leading cause of cancer-related deaths worldwide, with non-small cell lung cancer (NSCLC) making up the bulk of newly diagnosed cases (1). NSCLC is essentially untreatable, although many strategies have been proposed to improve patient survival (2). Most patients are diagnosed at an advanced stage, and half of them have distant metastatic disease at initial diagnosis, with poor prognosis (3). Thus, there is an urgent need for novel therapeutic targets and for advanced therapeutic strategies for the effective diagnosis and treatment of NSCLC.

With the development of genomic technologies, more and more molecular information including The Cancer Genome Atlas (TCGA) and Gene Expression Omnibus (GEO) represent a remarkable opportunity to analyze the gene expression data for the discovery of novel targets (4). The TCGA database has provided abundant resources for biological discovery due to the fact that it collects data related to over 30 types of human cancers (5). A recent study has reported that abnormal epigenetic modulation of mRNAs by microRNAs (miRNAs) may be associated with tumorigenesis (6). miRNAs are a class of endogenous RNAs of approximately 19-24 nucleotides (7), which play regulatory roles in cancers by targeting mRNAs for translational repression or degradation (8). Prognostic gene signatures for NSCLC have uncovered mutations in mRNAs and some aberrantly expressed miRNAs $(9,10)$. For instance, the expression of miR-34 is often altered in NSCLC tumor tissues. Of note, miR-34 targets key oncogenes involved in the tumorigenic process, such as $B C L 2, M Y C$, and $M E T(11,12)$. A recent simultaneous analysis of mRNA and miRNA 
expression profiles in NSCLC discovered 3,530 differentially expressed genes (DEGs) and 211 differentially expressed miRNAs (DEMs) in NSCLC when compared with matched para-carcinoma tissues (6). However, the combined potential of these DEGs and DEMs for effective molecular diagnosis of NSCLC still remains unclear.

In the present study, we re-analyzed the expression profiles of mRNAs and miRNAs in NSCLC in order to explore more specific molecular targets involved in the tumorigenesis of NSCLC, with the aim of establishing a combined diagnostic model based on several key genes and miRNAs. We perform a survival analysis for some key genes and miRNAs, followed by a multivariate logistic regression analysis.

\section{Materials and methods}

Expression profile dataset collection. Two datasets, GSE102286 and GSE101929 (13), containing the largest sample data sets of miRNAs and mRNAs with consistent samples (non-small cell lung cancer, tissue samples) collected in the past three years (since 2017) were downloaded from the GEO database. Briefly, GSE102286 is an miRNA expression profile dataset of 91 tumor tissue samples and 88 normal tissue samples from NSCLC patients obtained using the GPL23871 NanoString nCounter Human miRNA Expression Assay v1.6 platform. GSE101929 is an mRNA expression profile of 32 NSCLC tumor and 34 normal tissue samples from NSCLC patients, obtained using the GPL570 [HG-U133_Plus_2] Affymetrix Human Genome U133 Plus 2.0 Array platform. Moreover, the two sets of non-small cell lung cancer samples were confirmed to be lung adenocarcinoma. Additionally, lung adenocarcinoma miRNA and mRNA expression profiles were downloaded from the TCGA database, and information from 518 tumor and 58 adjacent tissue samples (control) were obtained. Of these samples, 490 had detailed clinical information.

Data preprocessing. After the CEL data were downloaded from the GEO database, the Oligo R software package (14) (version 1.34.0) was used for background correction of expression values and for uniform preprocessing of expression profile data, including format transformation, supplying missing values, background correction (MAS method), and data normalization by quantiles. The probes were annotated using the platform annotation file to remove the unmatched probes. If different probes mapped to the same gene or miRNA, the mean value of the different probes was used as the final expression value. The preprocessed data from TCGA, including the mRNA and miRNA counts, were downloaded.

Screening of differentially expressed genes/miRNAs. The expression matrices were divided into disease and control groups and were screened for DEMs and DEGs in the three datasets. Briefly, the processed data were analyzed using the paired samples t-test and corrected with the Benjamini/Hochberg method. An adjusted P-value $<0.05$ and $\mid \log 2$ fold change $(\mathrm{FC}) \mid>1$ were used as the threshold.

Venn analysis of DEMs and DEGs. The DEMs and DEGs that were common (overlapping in the Venn diagram) to both the
TCGA and GEO (GSE102286 for DEM, GSE101929 for DEG) datasets were selected for subsequent analyses.

miRNA and target gene. The miRWalk2.0 (15) tool was used to predict the miRNA target genes for all the overlapped DEMs. The commonly used databases (miRWalk (http://mirwalk.umm. uni-heidelberg.de/), miRanda (http://miranda.org.uk/), miRDB (http://mirdb.org/), miRNAMap (16), RNA22 (http://www. mybiosoftware.com/rna22-v2-microrna-target-detection.html) and Targetscan (http://www.targetscan.org/vert_72/)) were used for these predictions. The miRNA target pairs that were predicted by at least five databases were matched with the overlapped DEGs to obtain the DEM-DEG regulatory pairs. These regulatory relation pairs were visualized using Cytoscape (version 3.2.0) (17) and the topological properties of the network nodes were also analyzed.

Functional analysis of miRNAs and target genes. Based on the DEM-DEG interaction information, the miRNAs were subjected to Kyoto Encyclopedia of Genes and Genomes (KEGG) enrichment analysis (18) using the R software package clusterProfiler (19) (version 2.4.3). Results with $\mathrm{P}<0.05$ and count $>2$ were considered to be significantly enriched.

Moreover, functional enrichment analyses using Gene Ontology (GO) (20) and Kyoto Encyclopedia of Genes and Genomes (KEGG) were conducted on the DEGs of the DEM-DEG pairs using the gene functional enrichment analysis tool DAVID (version 6.8) $(21,22)$. P $<0.05$ and count $>2$ were used as the enrichment threshold.

Protein-protein interaction (PPI) network analysis. Using STRING (version 10.0) (23), the DEGs of the DEM-DEG pairs were analyzed to identify interactions between the DEGs. The PPI network was constructed using Cytoscape 3.2.0 and a PPI score of 0.4 (medium confidence). PPI network clustering modules were then analyzed using the Cytoscape plugin MCODE (version 1.4.2) (24) with a threshold of score $>10$. KEGG and GO enrichment analyses were then performed on the significant clustering modules.

Survival analysis of key miRNAs and DEGs. Using the miRNA matrix data from the regulatory network, the genes in the key TCGA modules and clinical information, the nodes were divided into high and low expression groups using the Survival R software package (25) (version 2.42-6) by median expression. Genes or miRNAs with a correlation coefficient of $\mathrm{P}<0.05$ were considered to influence survival prognosis, and the Kaplan-Meier (K-M) curve was drawn. Clinical information was analyzed based on the overall survival provided by TCGA.

Tissues samples from patients with NSCLC. This study was approved by the Ethics Committee of The Affiliated Huai'an Hospital of Xuzhou Medical University (Huai'an, China). A total of 32 pairs of NSCLC and normal tissue samples were collected from 45 NSCLC patients between January 2016 and December 2018 at the Affiliated Huai'an Hospital of Xuzhou Medical University. Each participant signed an informed consent form before the samples were collected. The clinical pathological parameters were as follows: 19 males 
and 13 females; average age was $54 \pm 12$ years; pathological types: Squamous cell carcinoma (14 cases), adenocarcinoma (18 cases), low differentiation (18 cases) and high differentiation (14 cases); there were 3 cases of stage I, 6 cases of stage II, 22 cases of stage III and 1 case of stage IV.

Cell lines and culture conditions. Additionally, human pulmonary alveolar epithelial cells (HPAEpiC; purchased from the Shanghai Guangdao Biotechnology Co., Ltd.) and three types of NSCLC cell lines (A549, H1299 and H460; purchased from the Cell Bank of the Chinese Academy of Science ) were used in this study. All cells were culture at $37^{\circ} \mathrm{C}$ in a humidified atmosphere of $5 \% \mathrm{CO}_{2}$ using Dulbecco's modified Eagle's medium (DMEM; Solarbio, Shanghai, China) with $10 \%$ fetal bovine serum (FBS; Gibco; Thermo Fisher Scientific, Inc.). Before verification of key miRNAs (those with the 18 highest degrees in the DEM-DEG regulatory network) and mRNAs (those with the five highest degrees in the significant modules) in the NSCLC tissue samples, the expression levels of these miRNAs and mRNAs were measured in HPAEpiC and three types of NSCLC cell lines using RT-qPCR. The mRNAs and miRNAs that had significantly differential expression in the HPAEpiC and at least two NSCLC cell lines were selected for verification in the tissue samples.

Real-time quantitative PCR (RT-qPCR). Total RNA was isolated from the cells and tissues using TRIzol reagent (Takara). Reverse transcription was conducted with the reverse transcription kit and qPCR kit (Takara). PCR was carried out using the SYBR Green qPCR Kit (Thermo Fisher Scientific, Inc.) with Applied Biosystems Viia7 Real-Time PCR System (Thermo Fisher Scientific, Inc.). Relative expression levels of mRNA were normalized to $\beta$-actin and calculated with the $2^{-\Delta \Delta \mathrm{Cq}}$ method. Primers of mRNA used in this study are listed in Table SI. The levels of miRNAs were measured by RT-qPCR using miDETECT A Track miRNA qRT-PCR Kit (RiboBio Co.). The primers for miRNAs and U6 small nuclear RNA were obtained from RiboBio Co. The sequences are covered by a patent. Analyses of miRNA expression were normalized to the expression of internal control U6 using the $2^{-\Delta \Delta \mathrm{Cq}}$ method.

Statistical analysis. Statistical analyses were performed using SPSS 23.0 (IBM Corp.). Differences between groups were analyzed by one-way analysis of variance (ANOVA) with the LSD test and were considered significant if the $\mathrm{P}$-value was $<0.05$.

Additionally, the Receiver Operating Characteristic (ROC) curves for key miRNAs and mRNAs were drawn using GraphPad Prism 7 (GraphPad Software, Inc.). Multivariate logistic regression analysis was then performed for key miRNAs and mRNAs with an area under the curve (AUC) $>0.7$ to explore the diagnosis value of the combined multi-index for NSCLC. The contingency table result was tested using the Hosmer-Lemeshow test for logistic regression analysis. A scatter diagram was drawn of the actual observed values (Observed) and the model-predicted values (Expected) and a linear trend line was fitted (calibration line) to obtain the calibration curve. The equation $\mathrm{y}=\mathrm{x}$ was used as the standard line equation. The closer the calibration line was to the standard line equation, the better the calibration capability of the model would be. In addition, the $\mathrm{C}$-index and ROC curves were used to verify the fitting effect of the model. A C-index $>0.9$ and AUC $>0.9$ indicated that the model had a good fitting effect.

Survival analysis was performed by Kaplan-Meier with log rank test, comparing high and low expression of each gene of interest (mean expression as the cut off value).

Network analysis for key miRNAs and mRNAs. After RT-qPCR and multivariate logistic regression analysis, reserved key miRNAs were subjected to miRNA-DEGs interaction analysis. The miRNA-DEG network was constructed using Cytoscape 3.2.0, and the topological properties of the network nodes were analyzed.

PPI network analysis of the DEGs in the miRNA-DEG interaction network was conducted with a low confidence PPI score of 0.15 . The modules in this network were analyzed using MCODE and scores $>5$ were regarded as significant. KEGG and GO enrichment analyses were carried out for the modules with significant PPI scores.

Furthermore, transcription factors (TFs) were predicted for the significant module genes using the Overrepresentation Enrichment Analysis (ORA) method in the WebGestalt toolkit (26). Cytoscape was used to construct the regulatory network from the top $10 \mathrm{TFs}$, with significant P-values.

\section{Results}

$D E M$ and DEG identification. After preprocessing the miRNA data, a total of 166 DEMs were identified from the TCGA database, of which 127 were significantly upregulated and 39 were significantly downregulated. A total of 79 DEMs were obtained from GSE102286, of which 51 were upregulated and 28 were downregulated. Venn diagram analysis identified 27 DEMs common to both datasets (Fig. 1A). Two of the 27 DEMs had opposite changes in expression in the two datasets; thus, the remaining 25 DEMs with consistent changes in expression (20 upregulated and five downregulated) were used for subsequent analyses.

From the mRNA data, a total of 4,888 DEGs consisting of 1,887 significantly upregulated DEGs and 3,001 significantly downregulated DEGs were obtained from TCGA. While 921 DEGs were obtained from GSE101929, consisting of 309 upregulated genes and 612 downregulated genes, Venn diagram analysis identified 789 common DEGs, of which 262 were upregulated and 527 were downregulated (Fig. 1B).

DEM-DEG regulatory network construction. Using the miRWalk2.0 database, 19 of the 25 overlapping DEMs were predicted to have miRNA target genes. The target DEGs were selected from the miRNA target pairs, finally producing 695 DEM-DEG relationship pairs. The 695 DEM-DEG pairs included 15 upregulated DEMs, 4 downregulated DEMs, 99 upregulated DEGs and 239 downregulated DEGs. A regulatory network was constructed with 357 nodes and 695 relationship pairs (Fig. 2A). The nodes with the highest degrees (top 20) are shown in Table I.

DEM-DEG functional enrichment. In the DEM-DEG pairs, eight miRNAs were significantly enriched in 59 KEGG 
A

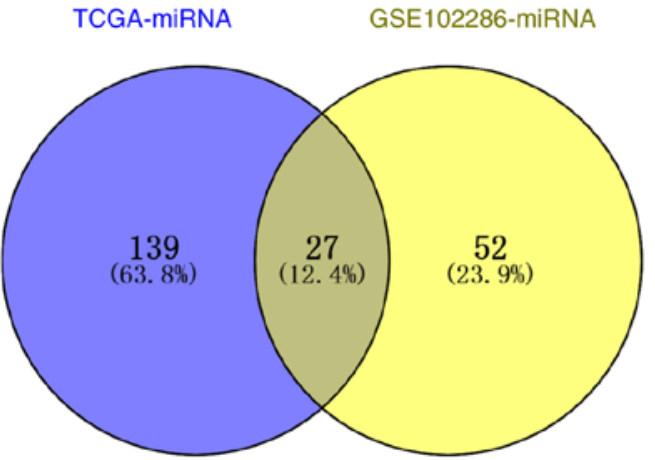

B

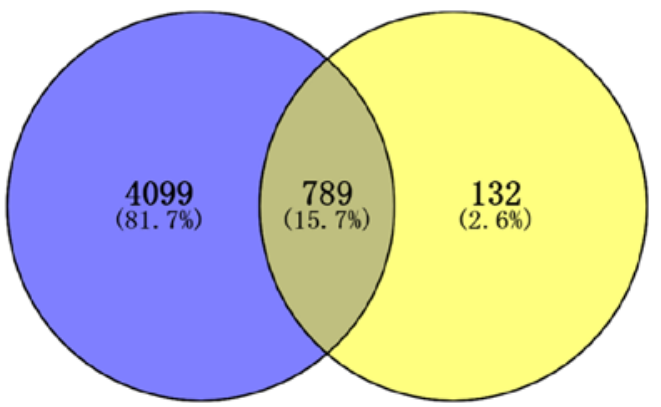

Figure 1. Venn diagrams of (A) miRNAs and (B) mRNAs from the GEO (GSE102286 and GSE101929) and The Cancer Genome Atlas (TCGA) databases.

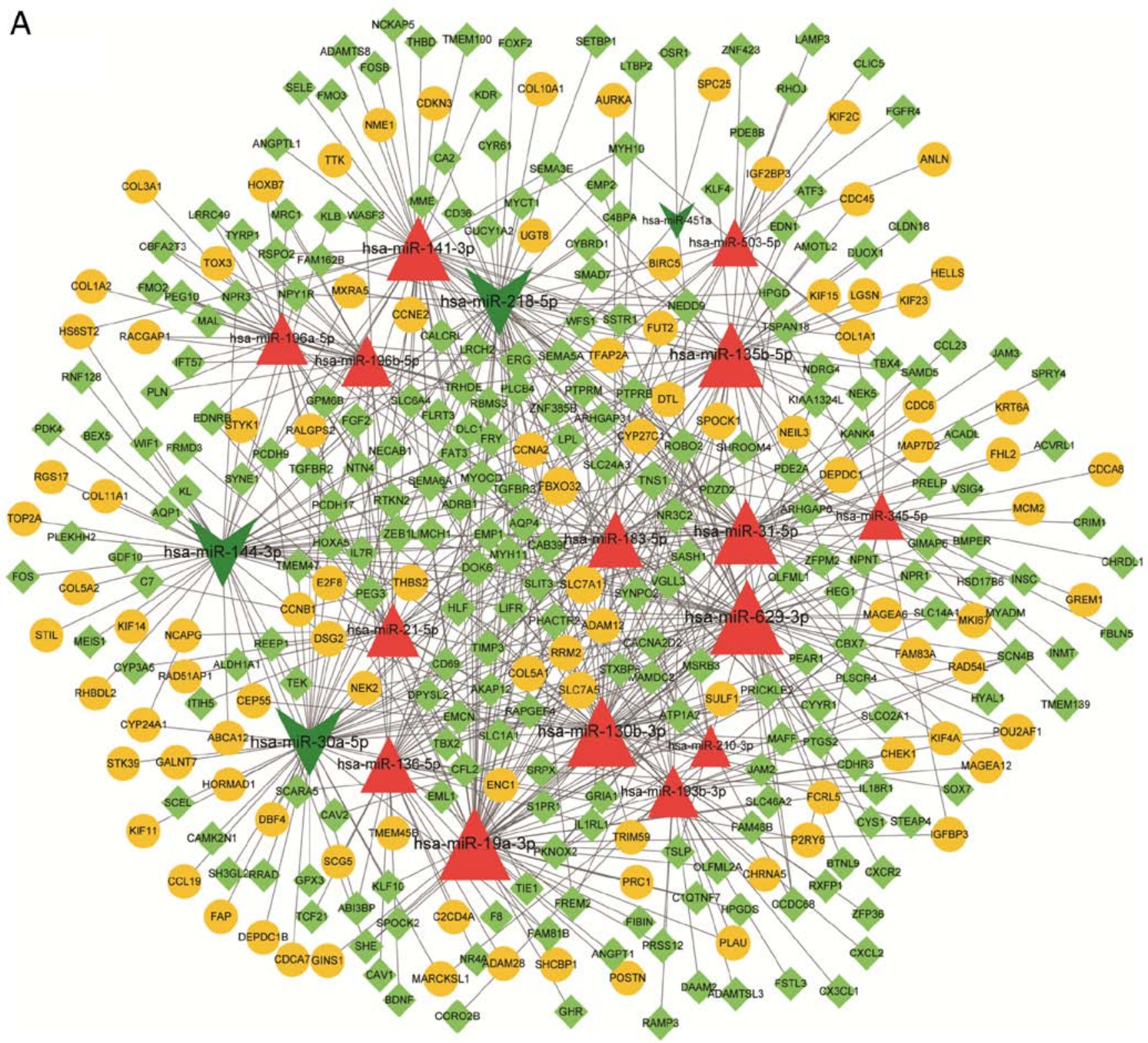

Figure 2. (A) miRNA target gene regulatory network for common differentially expressed miRNAs and mRNAs. Red triangles indicate upregulated miRNAs, dark green arrows indicate downregulated miRNAs, yellow circles indicate upregulated genes, and light green prisms indicate downregulated genes.

pathways, such as axon guidance, microRNAs in cancer, and TGF- $\beta$ signaling pathway. The top 5 most significant pathways are displayed in Fig. 3A.
The upregulated DEGs in DEM-DEG pairs were significantly enriched in 8 KEGG pathways, such as hsa04110: Cell cycle, hsa04512: ECM-receptor interaction, and hsa04115: 
B

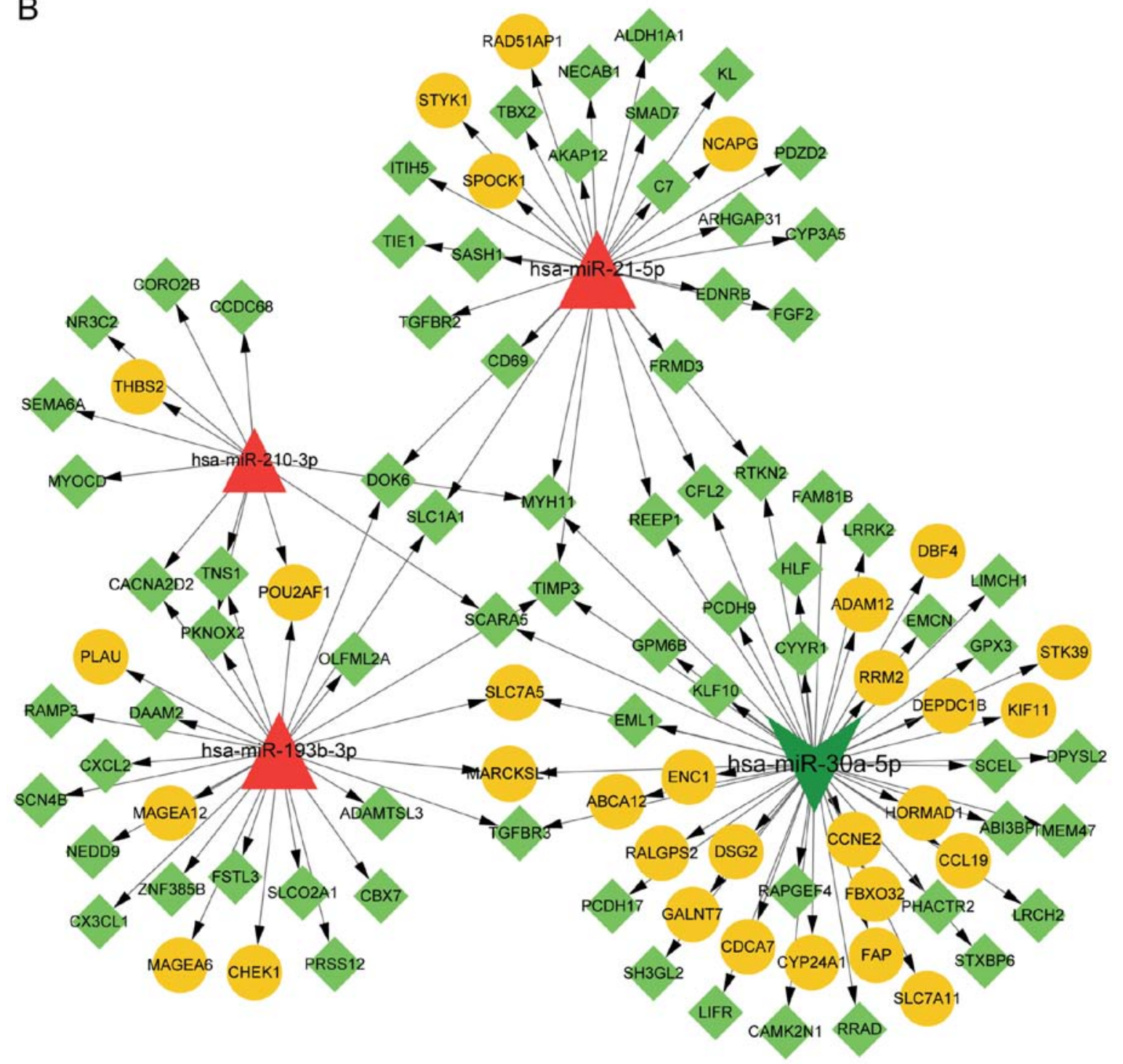

Figure 2. Continued. (B) miRNA target gene regulatory network for validated differentially expressed miRNAs and mRNAs. Red triangles indicate upregulated miRNAs, green arrows indicate downregulated miRNAs, yellow circles indicate upregulated genes, green prisms indicate downregulated genes, and the arrow indicates the regulation direction.

p53 signaling pathway, and enriched in 50 GO terms, such as cell division, and mitotic nuclear division. The downregulated genes were enriched in 8 KEGG pathways, such as hsa04024: cAMP signaling pathway, and hsa04360: Axon guidance, and enriched in $110 \mathrm{GO}$ terms. Due to the large number of GO results, we selected the top 20 terms for display (Fig. 3B-D).

PPI network construction. A PPI network was constructed based on the DEGs in the DEM-DEG pairs, as shown in Fig. 4A. The PPI network contained 228 nodes and 1,153 interaction pairs, including 79 upregulated DEGs and 149 downregulated DEGs. One module (score $=34.171$ ) was obtained, containing 36 nodes and 598 relationship pairs. The degrees of the nodes in the module are shown in Table II. Functional analyses revealed that the module genes were significantly enriched in three pathways (hsa04110: Cell cycle, hsa04115: p53 signaling pathway, and hsa05161: Hepatitis B) and 41 GO functions (such as cell division, and mitotic nuclear division) (Fig. 4B and C).
Survival analysis results. Based on the matrix data and clinical information obtained from the TCGA, we performed survival analysis for the 19 DEMs in the DEM-DEG pairs and 36 DEGs in the significant modules. The results showed a significant association between 34 DEGs including TOP $2 A$ (DNA topoisomerase II $\alpha$ ), CCNBI (cyclin B1), BIRC5 (baculoviral IAP repeat containing 5), TTK (TTK protein kinase) and two miRNAs (miR-21-5p and miR-31-5p) and patient prognosis (Fig. 5).

$R T$ - $q P C R$ verification results. After verification in the cell lines (Fig. 6A), 3 mRNAs (TOP2A, CCNB1 and BIRC5) and eight miRNAs (miR-19a-3p, miR-144-3p, miR-30a-5p, miR-31-5p, miR-21-5p, miR-193b-3p, miR-196a-5p and miR-210-3p) were further verified in tissue samples. As shown in Fig. 6B, the 3 mRNAs had significantly higher expression levels in cancer tissues than the controls. Additionally, miR-21-5p, miR-193b-3p, miR-31-5p and miR-210-3p were significantly upregulated while miR-30a-5p was significantly 

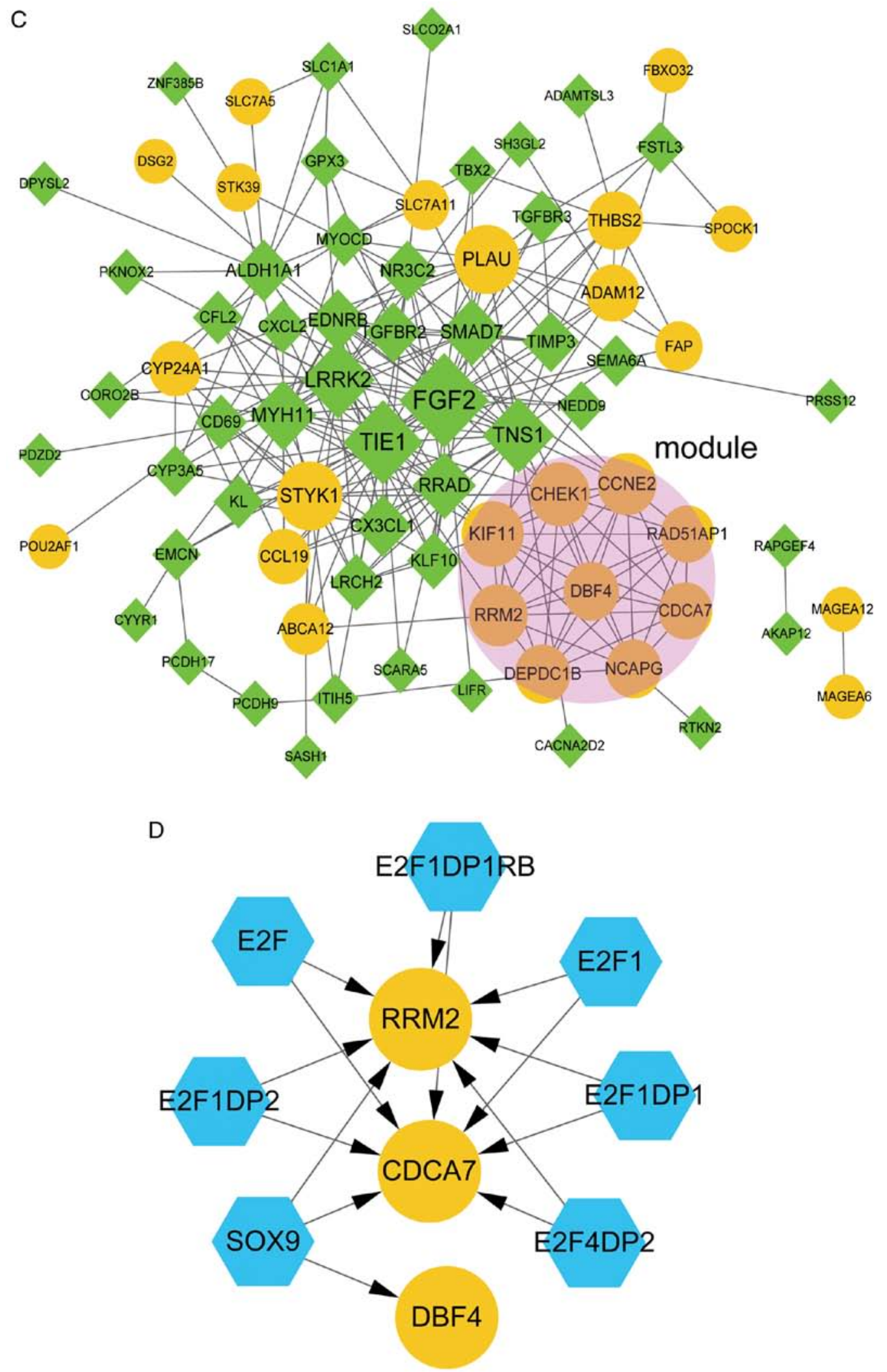

Figure 2. Continued. (C) PPI and module network diagram. Yellow circles represent upregulated genes, and green rhombuses represent downregulated genes. The area shaded in red is the module. Node size represents degree: The higher the degree, the larger the node. (D) Transcription factor-target regulatory network. Yellow circles represent upregulated genes, blue hexagons represent transcription factors, and node size represents degree. PPI, protein-protein interaction.

downregulated in cancer tissues in comparison with the control. These results were in accordance with 'DEM and DEG identification' results obtained by the bioinformatic analysis above.
Multivariate logistic regression analysis results. The ROC curves of the three mRNAs (TOP2A, CCNB1, and BIRC5) and five miRNAs (miR-21-5p, miR-193b-3p, miR-31-5p, miR-210-3p and miR-30a-5p) are shown in Fig. 6C. After 
Table I. Degree nodes in the miRNA target gene regulatory network (top 20).

\begin{tabular}{lllll}
\hline Nodes & Description & Degree & Nodes & Description \\
\hline miR-629-3p & Up-miRNA & 60 & miR-183-5p & Up-miRNA \\
miR-19a-3p & Up-miRNA & 57 & miR-21-5p & Up-miRNA \\
miR-130b-3p & Up-miRNA & 53 & miR-193b-3p & Up-miRNA \\
miR-144-3p & Down-miRNA & 52 & miR-196a-5p & Up-miRNA \\
miR-30a-5p & Down-miRNA & 52 & miR-196b-5p & Up-miRNA \\
miR-218-5p & Down-miRNA & 51 & miR-503-5p & Up-miRNA \\
miR-135b-5p & Up-miRNA & 47 & miR-345-5p & Up-miRNA \\
miR-31-5p & Up-miRNA & 46 & miR-210-3p & Up-miRNA \\
miR-141-3p & Up-miRNA & 45 & TNS1 & Down-gene \\
miR-136-5p & Up-miRNA & 34 & DOK6 & Down-gene
\end{tabular}

up-miRNA, upregulated miRNA; down-miRNA, downregulated miRNA; down-gene, downregulated gene. TNS1, tensin 1; DOK6, docking protein 6.
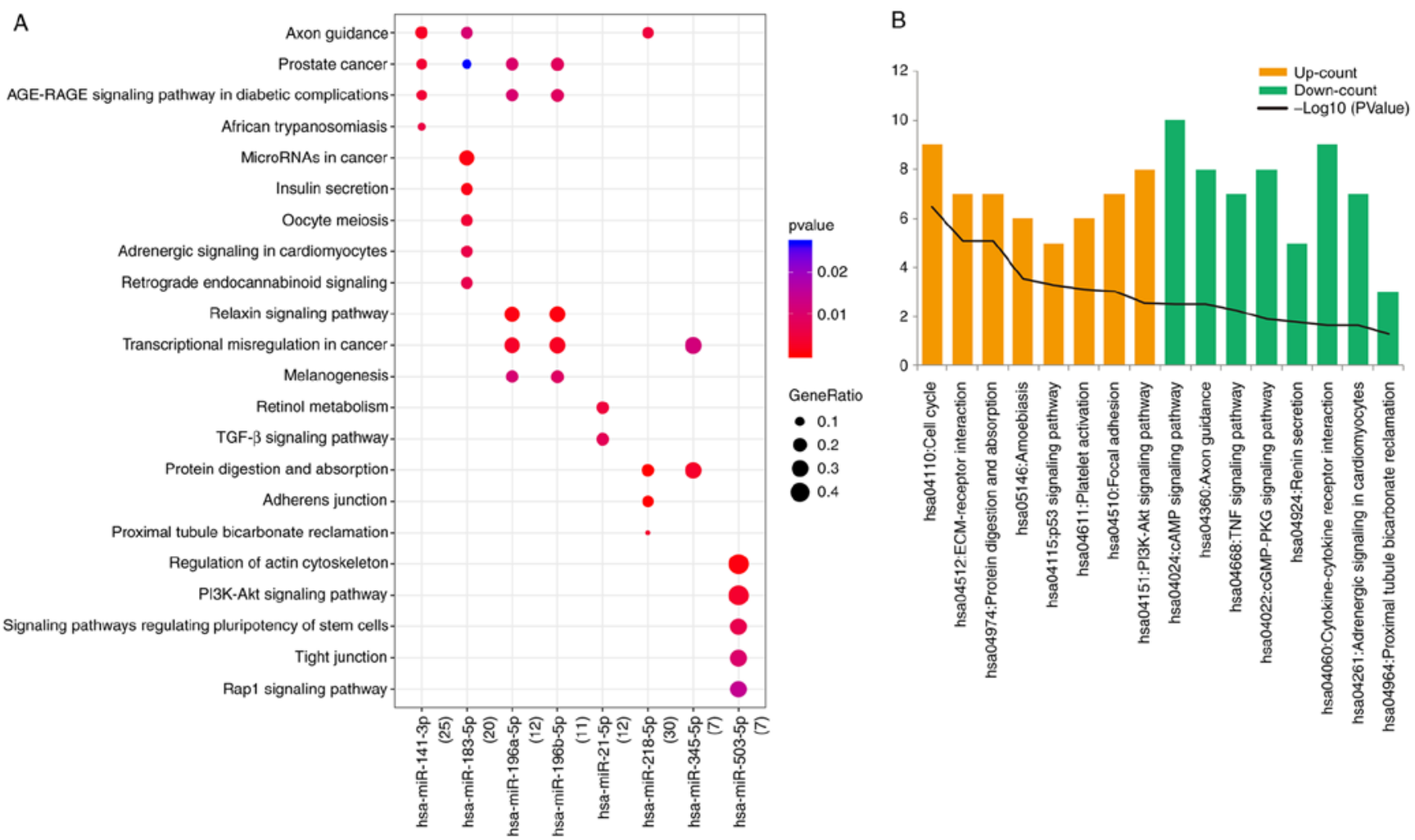

Figure 3. (A) KEGG pathway bubbles enriched by differentially expressed miRNAs. (B) KEGG pathways enriched by the target genes. GO, Gene Ontology; KEGG, Kyoto Encyclopedia of Genes and Genomes.

analysis of the ROC curves, miR-30a-5p showed low diagnostic value for NSCLC (AUC $=0.665)$, while the remaining miRNAs and all the mRNAs had high diagnostic values (AUC $>0.7$ ) in the TCGA dataset. Thus, TOP $2 A, C C N B 1$, BIRC5, miR-21-5p, miR-193b-3p, miR-210-3p and miR-31-5p were selected for multivariate logistic regression analysis. The results of the multivariate logistic regression analysis of the selected mRNAs and miRNAs in the TCGA dataset and 32 pairs of tissue samples, and the ROC curves for combined diagnosis are shown in Tables SII-SV and Fig. 7, respectively.
Network analysis for three mRNAs and four miRNAs. Based on the four selected miRNAs, a miRNA-DEG regulatory network was constructed with 107 nodes (3 upregulated miRNAs, 1 downregulated miRNAs, 31 upregulated DEGs and 72 downregulated DEGs) and 120 interaction pairs (Fig. 2B).

The PPI network constructed from the 31 upregulated DEGs and 72 downregulated DEGs consisted of 75 nodes and 248 interaction pairs. From this network, one module (score=9) was extracted, which included nine nodes and 36 interaction pairs (Fig. 2C). The genes in the module were involved in two pathways (hsa04115: p53 signaling pathway, and hsa04110: 
C

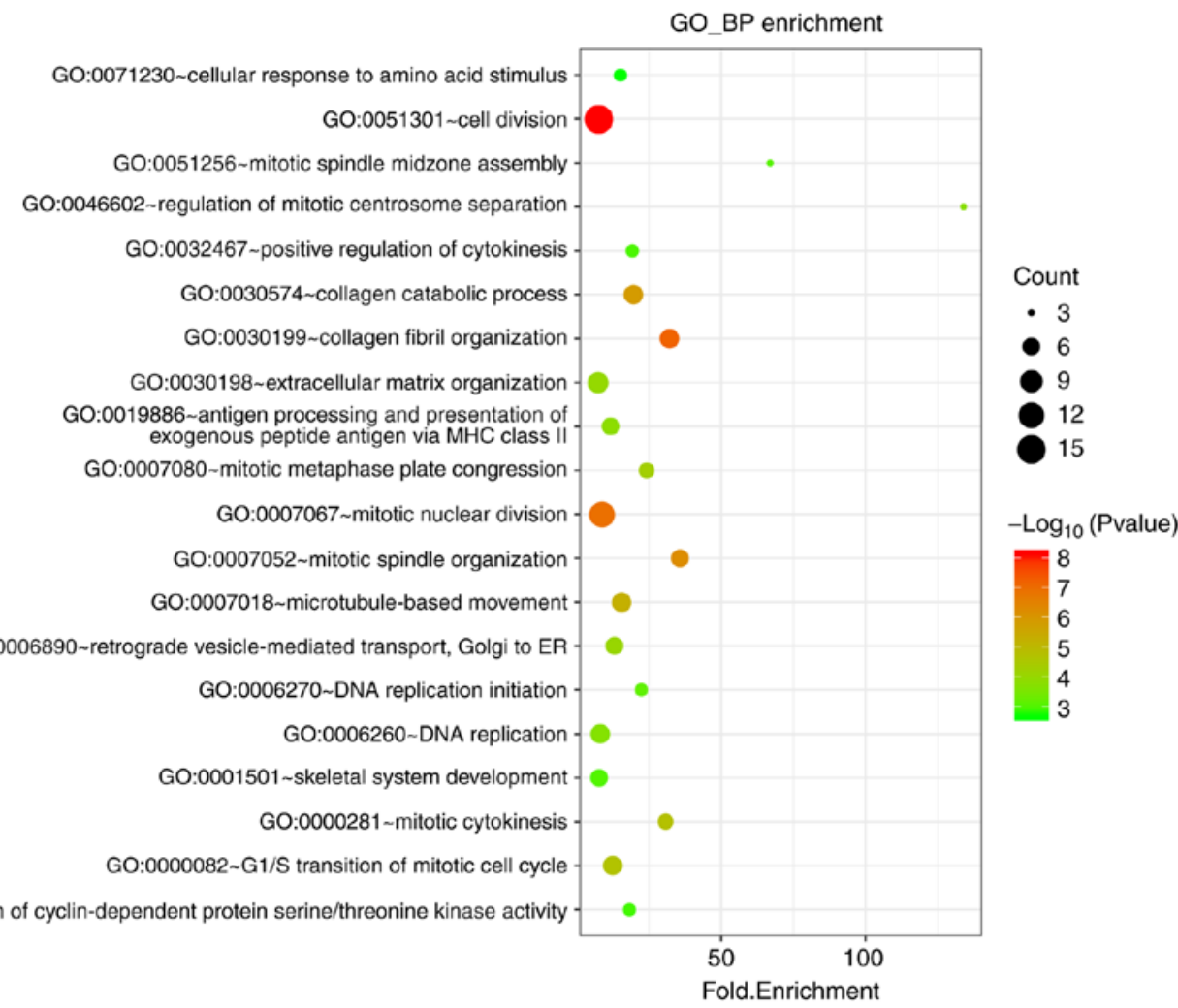

D

GO BP enrichment GO:0070374-positive regulation of ERK1 and ERK2 cascade GO:0050919 negative chemotaxis GO:0048661 positive regulation of smooth muscle cell proliferation GO:0045766 positive regulation of angiogenesis GO:0035810 positive regulation of urine volume GO:0031623 receptor internalization GO:0030198 extracellular matrix organization GO:0016525 negative regulation of angiogenesis

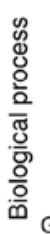
GO:0010595 positive regulation of endothelial cell migration GO:0008217 regulation of blood pressure GO:0007411 axon guidance

GO:0007399 nervous system development GO:0007179 transforming growth factor beta receptor signaling pathway GO:0007166 cell surface receptor signaling pathway GO:0007165 signal transduction GO:0001701 in utero embryonic development GO:0001657 ureteric bud development GO:0001570 vasculogenesis GO:0001525 angiogenesis GO:0000122 negative regulation of transcription from RNA polymerase II promoter

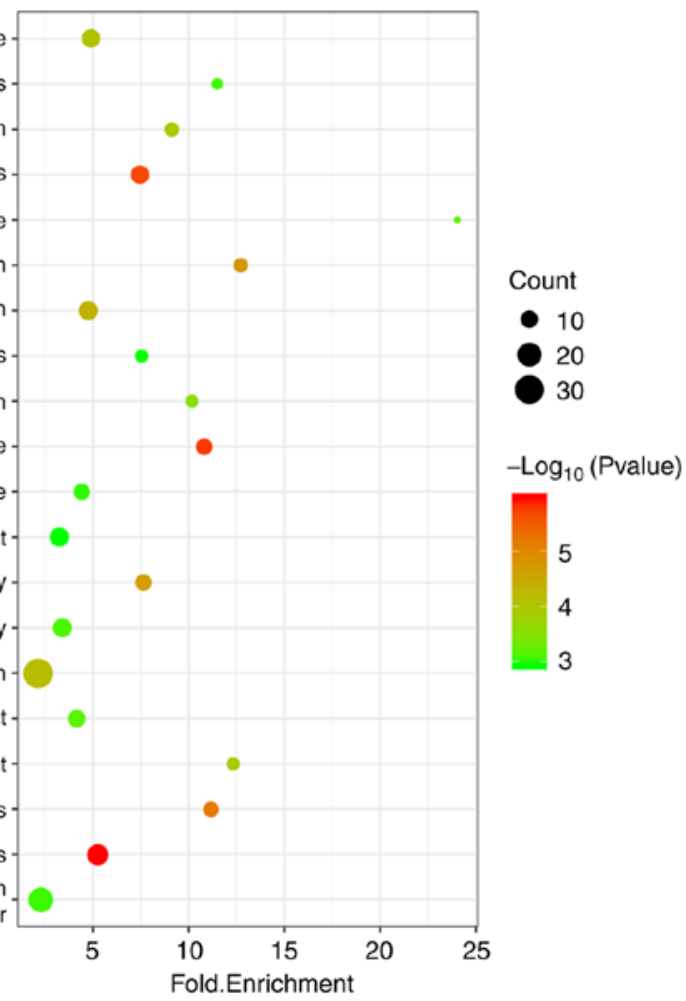

Figure 3. Continued. (C) GO function bubbles enriched by upregulated target genes. (D) GO function bubbles enriched by downregulated target genes. GO, Gene Ontology; KEGG, Kyoto Encyclopedia of Genes and Genomes.

cell cycle) and $5 \mathrm{GO}$ functions, including cell division, and the G1/S transition of the mitotic cell cycle.

Furthermore, 7 TFs, such as SRY-box 9 (SOX9), were predicted for the genes in the module, involving 15 regulatory relationship pairs (Fig. 2D).

\section{Discussion}

In the present study, 25 overlapped differentially expressed miRNAs (DEMs) and 789 overlapped differentially expressed genes (DEGs) were identified, which were then used to 



C

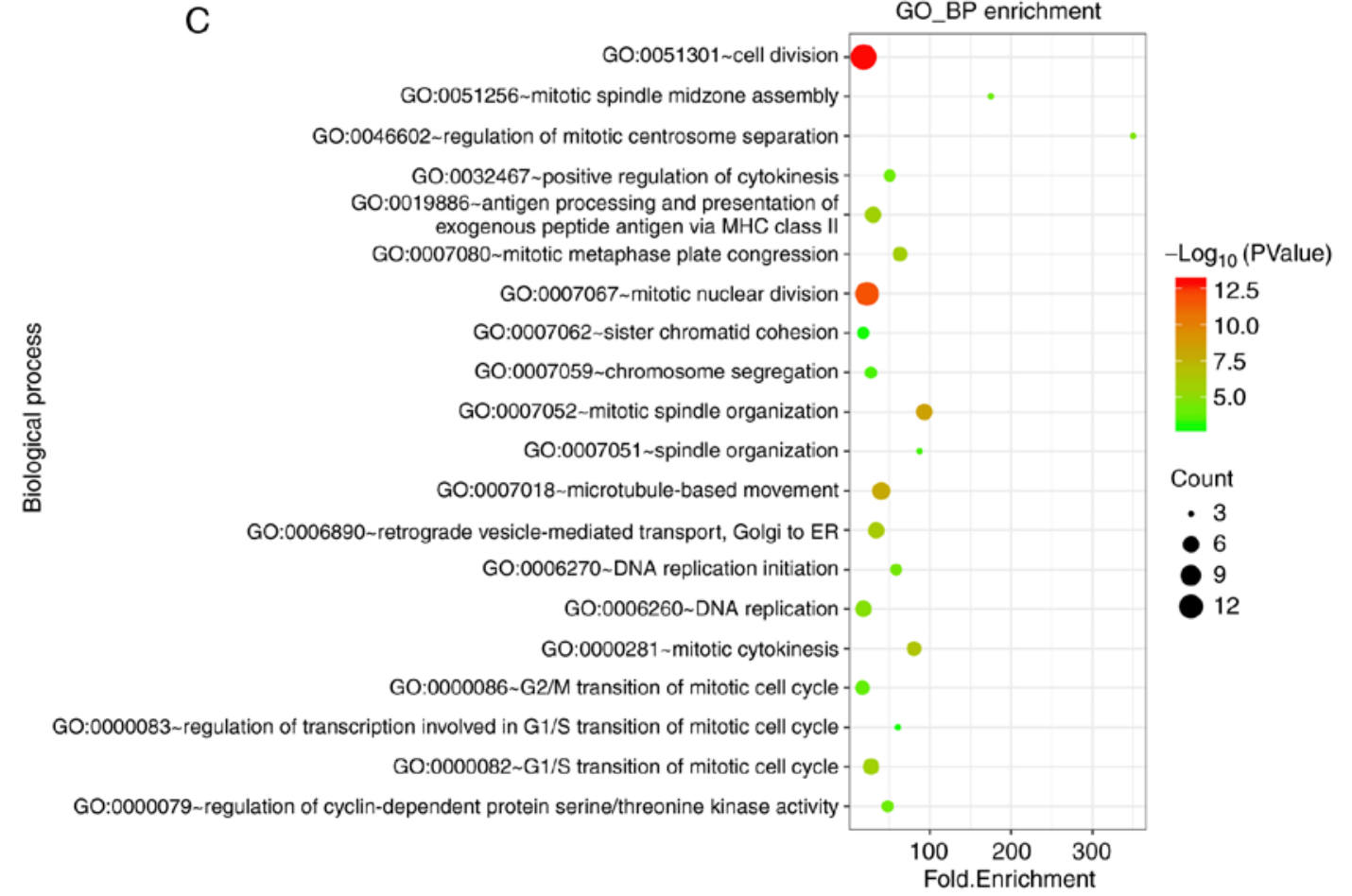

Figure 4. (A) PPI and module network diagram. Yellow circles represent upregulated genes, and light green rhombuses represent downregulated genes. The shaded part is the module. Node size represents the degree: The higher the degree, the larger the node. (B) KEGG pathways enriched by module nodes. (C) GO functions enriched by module nodes. PPI, protein-protein interaction; GO, Gene Ontology; KEGG, Kyoto Encyclopedia of Genes and Genomes.

construct a DEM-DEG regulatory network and protein-protein interaction (PPI) network module. Survival analysis of the 19 DEMs in the DEM-DEG regulatory network and the 36 DEGs in the PPI network module revealed that 34 DEGs (including TOP2A, CCNB1, BIRC5, TTK) and two miRNAs (miR-21-5p and miR-31-5p) were significantly associated with patient prognosis. Moreover, RT-qPCR analysis of the top 18 DEMs in the DEM-DEG regulatory network and the top 5 DEGs in the PPI network module identified 3 DEGs and 5 DEMs that had expression profiles consistent with the bioinformatic analysis results. Finally, multivariate logistic regression analysis suggested that TOP $2 A, C C N B 1, B I R C 5$, miR-21-5p, miR-193b-3p, miR-210-3p and miR-31-5p could be used together for the diagnosis of non-small cell lung cancer (NSCLC).

TOP2A (DNA topoisomerase II $\alpha$ ) had the highest degree in the significant module. It encodes a DNA topoisomerase II, which controls the topological state of the DNA during transcription. It has been reported that topoisomerase II is involved in DNA synthesis and cell proliferation, both of which are common targets of antitumor drugs (27). Recently, Terashima et al (28) demonstrated that in patients with stage II/III gastric cancer, the TOP $2 A$ level in primary tumors is associated with a higher risk of hematogenous recurrence of cancer. Importantly, a previous study suggested that $T O P 2 A$ expression is related 
Table II. Degree values of the module nodes.

\begin{tabular}{|c|c|c|c|c|c|}
\hline Nodes & Description & Degree & Nodes & Description & Degree \\
\hline TOP $2 A$ & Up-gene & 53 & MKI67 & Up-gene & 37 \\
\hline$C C N B 1$ & Up-gene & 43 & KIF4A & Up-gene & 37 \\
\hline$R A C G A P 1$ & Up-gene & 43 & PRC1 & Up-gene & 36 \\
\hline BIRC5 & Up-gene & 41 & $A N L N$ & Up-gene & 36 \\
\hline$T T K$ & Up-gene & 41 & CDCA 8 & Up-gene & 36 \\
\hline$C D K N 3$ & Up-gene & 40 & $N E K 2$ & Up-gene & 36 \\
\hline CHEK1 & Up-gene & 40 & $K I F 2 C$ & Up-gene & 36 \\
\hline KIF 11 & Up-gene & 40 & KIF 14 & Up-gene & 36 \\
\hline CCNA2 & Up-gene & 40 & $M C M 2$ & Up-gene & 35 \\
\hline KIF 23 & Up-gene & 40 & $R A D 51 A P 1$ & Up-gene & 35 \\
\hline$N C A P G$ & Up-gene & 40 & $S H C B P 1$ & Up-gene & 34 \\
\hline CEP55 & Up-gene & 40 & $D E P D C 1$ & Up-gene & 33 \\
\hline$R R M 2$ & Up-gene & 39 & $H E L L S$ & Up-gene & 33 \\
\hline$A U R K A$ & Up-gene & 39 & $R A D 54 L$ & Up-gene & 31 \\
\hline$C D C 45$ & Up-gene & 39 & STIL & Up-gene & 29 \\
\hline CDC6 & Up-gene & 38 & $S P C 25$ & Up-gene & 29 \\
\hline KIF 15 & Up-gene & 38 & NEIL3 & Up-gene & 27 \\
\hline$D T L$ & Up-gene & 38 & CCNE2 & Up-gene & 26 \\
\hline
\end{tabular}

Up-gene, upregulated gene. TPO2A, DNA topoisomerase II $\alpha ; C C N B 1$, cyclin B1; RACGAP1, Rac GTPase activating protein 1; BIRC5, baculoviral IAP repeat containing 5; TTK, TTK protein kinase; $C D K N 3$, cyclin dependent kinase inhibitor 3; CHEK1, checkpoint hinase 1; KIF11, kinesin family member $11 ; C C N A 2$, cyclin A2; KIF23, kinesin family member $23 ; N C A P G$, non-SMC condensin I complex subunit G; $C E P 55$, centrosomal protein 55; RRM2, ribonucleotide reductase regulatory subunit M2; AURKA, Aurora kinase A; $C D C 45$, cell division cycle 45; CDC6, cell division cycle 6; KIF15, kinesin family member 15; DTL, Denticleless E3 ubiquitin protein ligase homolog; MKI67, marker of proliferation Ki-67; KIF4A, kinesin family member 4A; PRC1, protein regulator of cytokinesis 1; ANLN, Anillin actin binding protein; $C D C A 8$, cell division cycle associated 8; NEK2, NIMA related kinase 2; KIF2C, kinesin family member 2C; KIF 14, kinesin family member 14; $M C M 2$, minichromosome maintenance complex component 2; RAD51AP1, RAD51 associated protein 1; SHCBP1, SHC binding and spindle associated 1; DEPDC1, DEP domain containing 1; HELLS, helicase, lymphoid specific; RAD54L, RAD54 like; STIL, STIL centriolar assembly protein; SPC25, SPC25 component of NDC80 kinetochore complex; NEIL3, Nei like DNA glycosylase 3; CCNE2, cyclin E2.

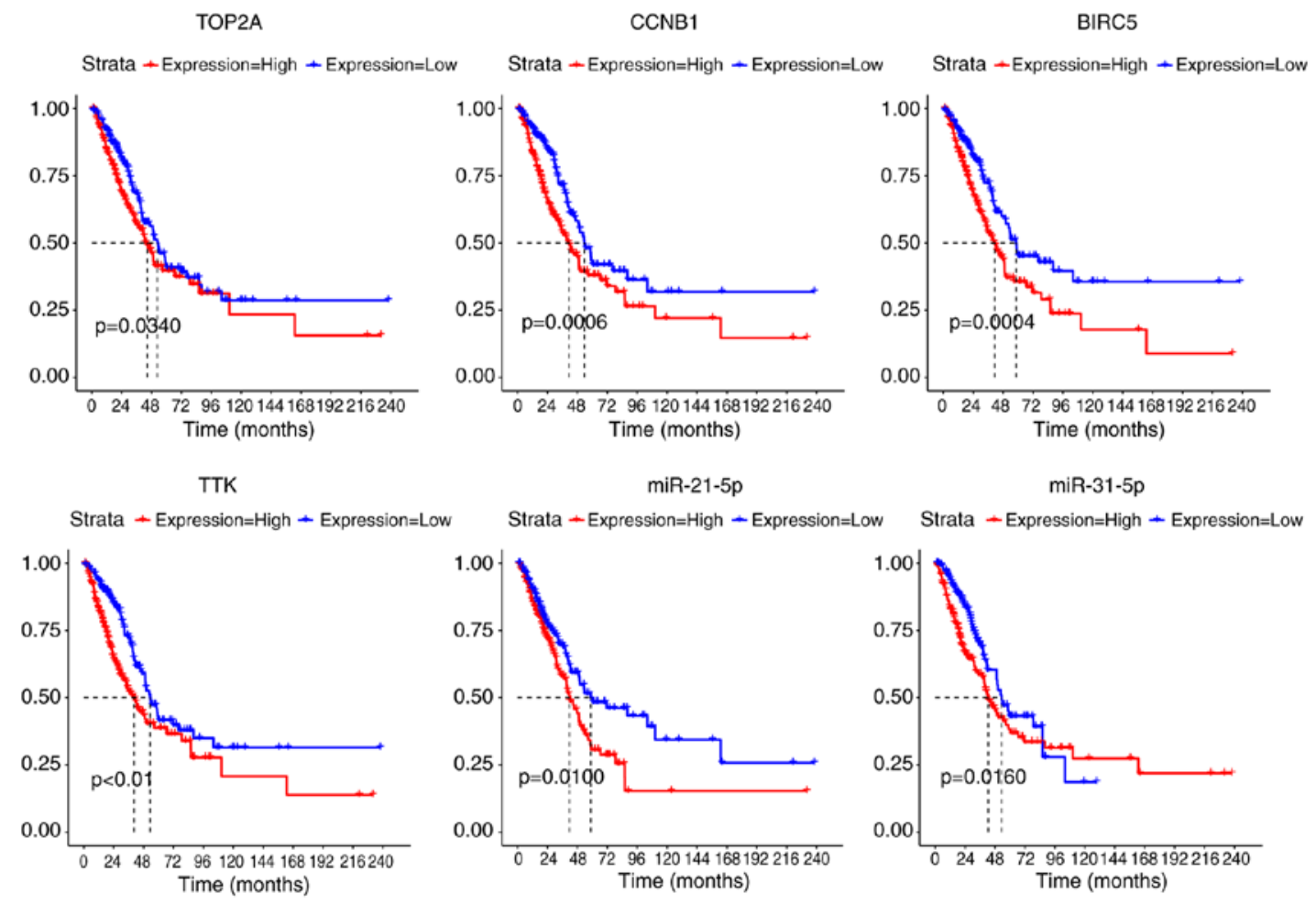

Figure 5. Survival curves for TOP2A, CCNB1, BIRC5, TTK, miR-21-5p and miR-31-5p. TPO2A, DNA topoisomerase II $\alpha$; CCNB1, cyclin B1; BIRC5, baculoviral IAP repeat containing $5 ;$ TTK, TTK protein kinase. 
A
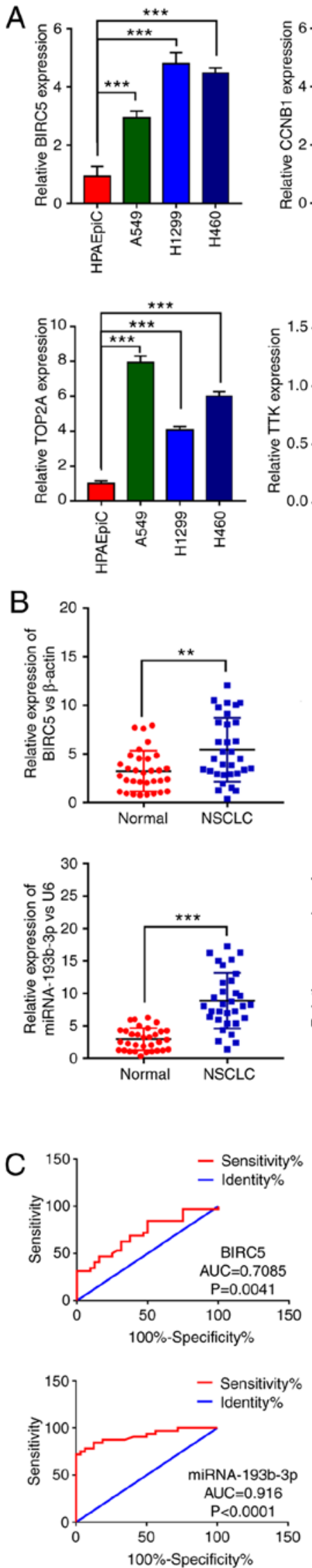
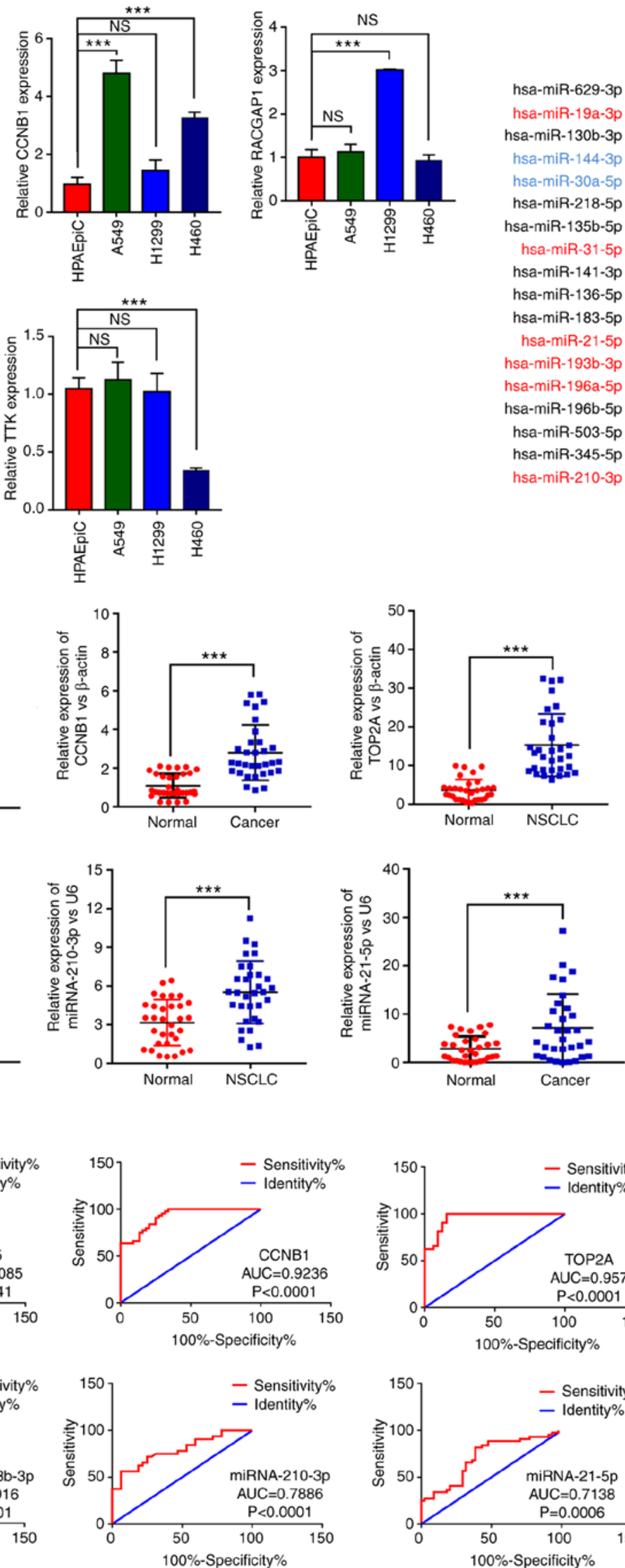
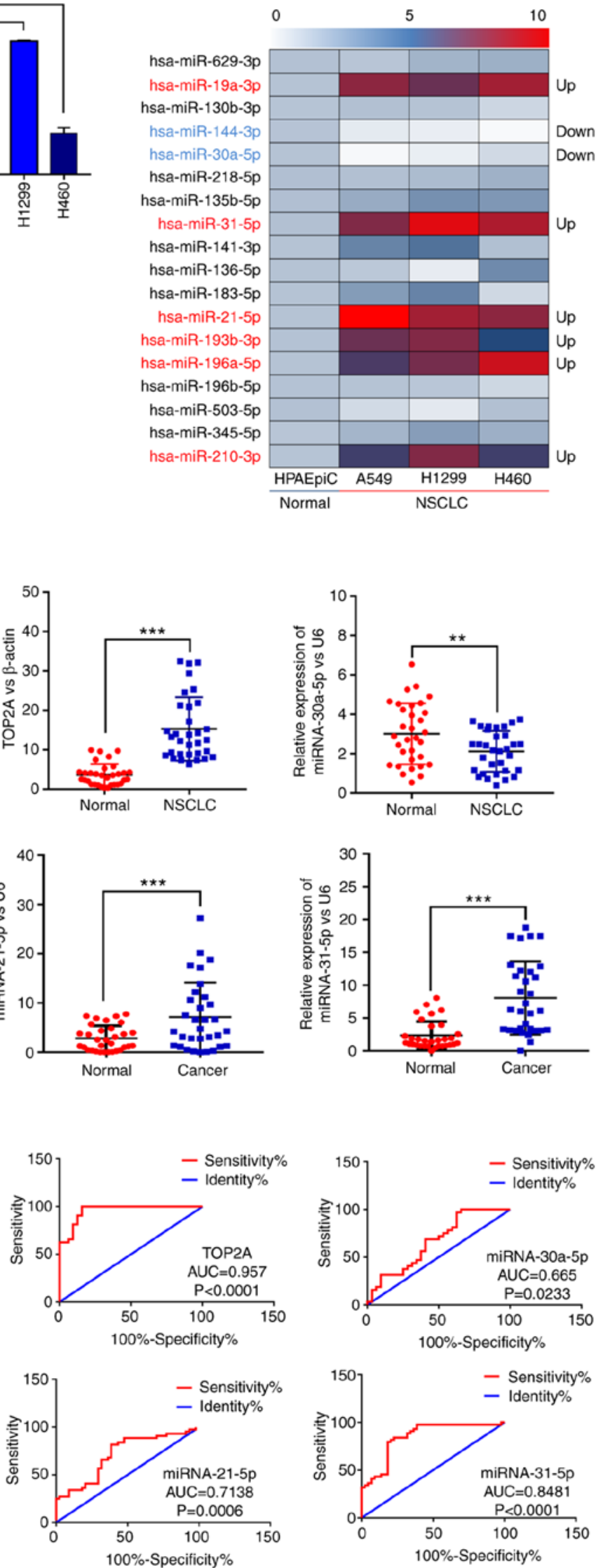

Figure 6. (A) Relative expression levels of five DEGs and 18 DEMs in NSCLC and normal cells. (B) The relative expression levels of TOP2A, CCNB1, BIRC5, miR-30a-5p, miR-21-5p, miR-193b-3p, miR-31-5p and miR-210-3p in cancer and normal tissues. (C) The ROC curves of TOP2A, CCNB1, BIRC5, miR-30a-5p, miR-21-5p, miR-193b-3p, miR-31-5p and miR-210-3p. DEGs, differentially expressed genes; DEMs, differentially expressed miRNAs; NSCLC, non-small cell lung cancer; TPO2A, DNA topoisomerase II $\alpha$; CCNB1, cyclin B1; RACGAP1, Rac GTPase activating protein 1; BIRC5, baculoviral IAP repeat containing 5; TTK, TTK protein kinase. 
A

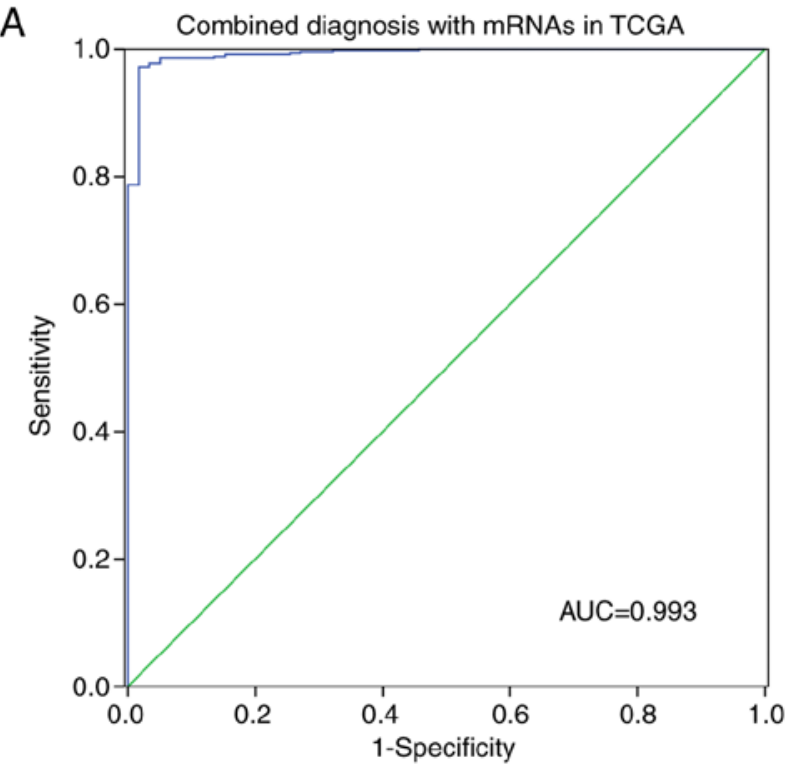

C

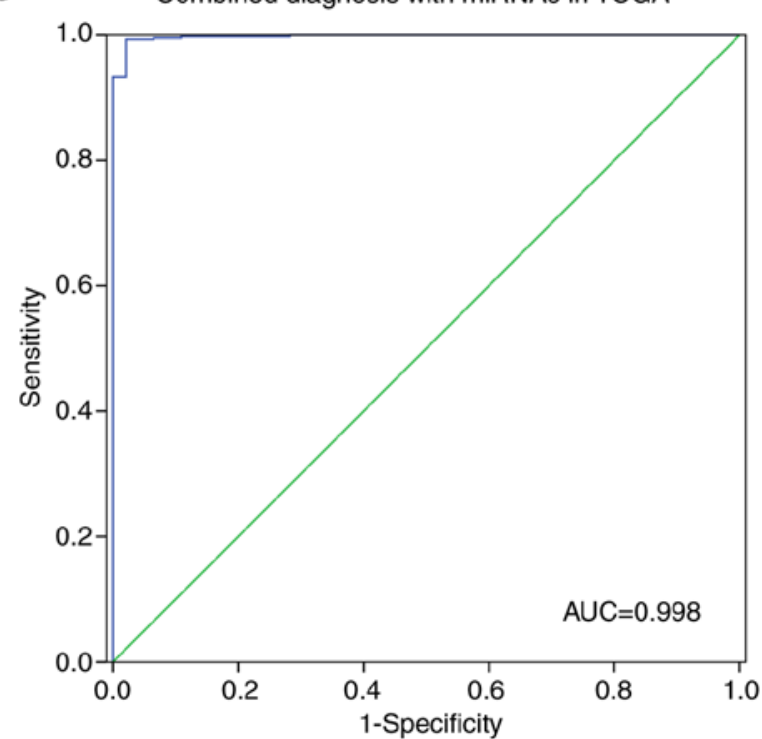

B

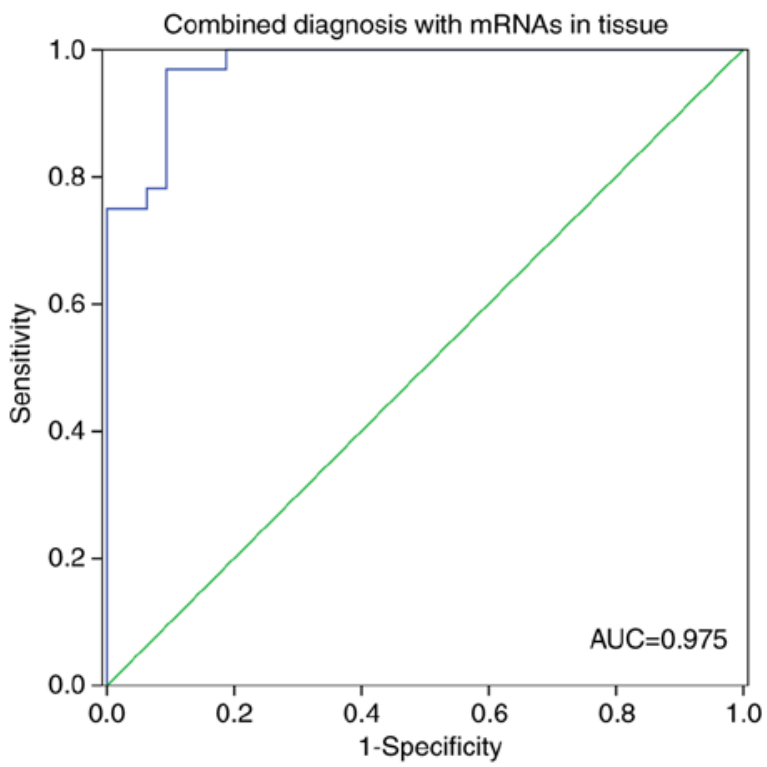

D

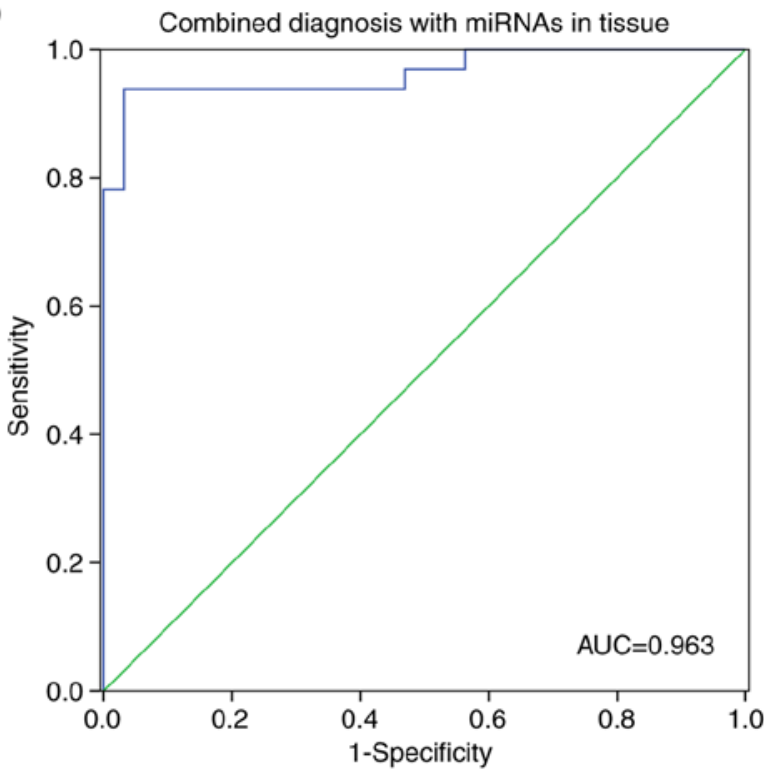

Figure 7. ROC curves for combined diagnosis with (A and B) 4 miRNAs (miR-21-5p, miR-193b-3p, miR-31-5p, miR-210-3p) and (C and D) 3 mRNAs (TOP2A, $C C N B 1, B I R C 5)$ in the TCGA dataset and 32 pairs of tissue samples, respectively. ROC, receiver operating characteristic; AUC, area under the ROC curve; TCGA, The Cancer Genome Atlas; TPO2A, DNA topoisomerase II $\alpha$; CCNB1, cyclin B1; BIRC5, baculoviral IAP repeat containing 5.

to tumor cell proliferation and clinicopathological parameters in lung adenocarcinoma (29). In this study, TOP $2 A$ was upregulated in non-small cell lung cancer (NSCLC) cell lines and tissues. In addition, TOP $2 A$ was predicted to be a prognostic factor and had high diagnostic value in NSCLC. Based on a review of previous studies and the results of this study, TOP $2 A$ may serve as an important diagnostic and therapeutic target in NSCLC.

CCNB1 (cyclin B1), a cell cycle regulator, which combines with cyclin-dependent kinase 1 (CDK1) to form a complex, had the second highest degree in the module. The CCNB1-CDK1 complex has been reported to be a key factor controlling the $\mathrm{G} 2 / \mathrm{M}$ phase of the cell cycle (30). In line with that report, our functional analysis revealed that $C C N B 1$ was involved in the hsa04110: Cell cycle pathway. This study also supports previous observations where $C C N B 1$ has been shown to be overexpressed in many cancers, including lung cancers $(31,32)$. Notably, in the present study, CCNB1 was shown to be enriched in the hsa04115: p53 signaling pathway. Human p53 is a 393-amino acid nuclear protein that acts as a transcription factor (TF) and tumor suppressor (33). Activated p53 is known to induce growth arrest, which allows cells to repair damage. Moreover, p53 is frequently inactivated during human carcinogenesis (34). Thus, CCNB1 may be involved in the progression of NSCLC via the cell cycle and p53 signaling pathways.

BIRC5 (baculoviral IAP repeat containing 5) is a unique member of the inhibitor-of-apoptosis gene family (35), which is usually present in transformed cells, fetal development, as well as tumors, but absent in most normally differentiated adult tissues (36). It has previously been reported that BIRC5 can inhibit apoptosis via both the mitochondrial and the death-receptor pathways (37). Additionally, it acts as a regulator of cell division. In line with the previous studies, the functional analysis 
conducted in this study also suggests that BIRC5 is associated with cell division and mitotic nuclear division. Furthermore, circulating antibodies to BIRC5 have been shown to be elevated in many malignant tumors, including NSCLC (38). Therefore, upregulated BIRC5 may serve as a useful biomarker for the prognostic assessment and diagnosis of NSCLC.

miR-30a-5p is a member of the miR-30 family, which plays a key role in several physiological and pathological processes, including differentiation, development, and apoptosis (39). The results of the current study are consistent with earlier observations, which have shown that this miRNA is significantly downregulated in NSCLC tissues (40). Interestingly, three target genes of miR-30a-5p, including ribonucleotide reductase subunit M2 (RRM2), cell division cycle associated 7 (CDCA7), and DBF4 zinc finger (DBF4), were predicted to be transcriptionally controlled by the TF SOX9. High levels of SOX9 are correlated with poor survival of NSCLC patients (41). We speculate that there is a regulatory relationship between miR-30a-5p and SOX9 in the pathogenesis of NSCLC, which needs to be further investigated. Remarkably, a recent study by Świtlik et al (42) revealed that miR-30a-5p together with miR-210-3p can distinguish NSCLC tissues from normal adjacent tissues. Moreover, they reported a high diagnostic value for a combination of the two miRNAs through ROC analysis and also suggested the combination of miR-30a-5p with miR-210-3p as an independent biomarker for NSCLC.

miR-21 has been previously reported to be a key biomarker for the early detection of lung cancer, and overexpression of miR-21 is implicated in a worse prognosis for lung cancer patients (43). In this study, high expression of miR-21-5p was likewise correlated with poor prognosis of NSCLC patients. KEGG pathway analysis showed that miR-21-5p was enriched in the TGF- $\beta$ signaling pathway. This pathway has been suggested to facilitate tumor metastasis in lung adenocarcinoma (44). In the miR-21-5p associated DEM-DEG regulatory network, the non-SMC condensin I complex subunit $\mathrm{G}(N C A P G)$ was one of its targets and is involved in $\mathrm{GO}$ function related to cell division. A recent study suggested that $N C A P G$ promotes tumor proliferation by regulating the $\mathrm{G} 2 / \mathrm{M}$ phase in lung adenocarcinoma (45). Thus, we speculate that miR-21-5p may play a role in NSCLC progression through the TGF- $\beta$ signaling pathway or by regulating $N C A P G$.

A recent study suggested that miR-193b has both tumor-suppressor and oncogenic functions in human cancers. Lower levels of miR-193b expression were detected in NSCLC cells when compared to that in non-cancerous cells (46). In contrast, using RT-qPCR our study verified that miR-193b-3p was upregulated in NSCLC cells and tissues compared to the controls. These results corroborated with our bioinformatic analysis. Although these findings contradict previous results, we can conclude that the aberrant expression of miR-193b-3p may serve as a biomarker of NSCLC.

However, there were some limitations to our study. First, although the data set contained two databases with a large sample size, it still had no absolute universal significance and could only be of reference significance to a certain extent. Second, the in-depth analysis of DEGs was limited to those with regulatory relationship with DEMs, and could have missed various gene studies with other roles. Our future research will focus on verifying the clinical utility of these genes and miRNAs in larger samples.

In conclusion, our study combined mRNA and miRNA expression profiles in NSCLC to identify more diagnostic and therapeutic targets for NSCLC. TOP2A, CCNB1, BIRC5, miR-21-5p, miR-193b-3p, miR-210-3p and miR-30a-5p may serve as biomarkers and can be combined for the diagnosis of NSCLC.

\section{Acknowledgements}

Not applicable.

\section{Funding}

This study was supported by grants from the Natural Science Research Program of Huai'an City (HAB201813).

\section{Availability of data and materials}

The data used to support the findings of this study are included within the article and supplementary files.

\section{Authors' contributions}

YW, SC and YulZ conceived and designed the experiments. JZ and DL performed the experiments. YueZ and ZD analyzed the data. JZ and DL drafted the manuscript. All authors read and approved the final manuscript.

\section{Ethics approval and consent to participate}

This study was approved by the Ethics Committee of the Affiliated Huai'an Hospital of Xuzhou Medical University (Huai'an, China). Each participant signed an informed consent form before the samples were collected.

\section{Patient consent for publication}

Not applicable.

\section{Competing interests}

The authors declare that they have no competing interests.

\section{References}

1. Kasinski AL, Kelnar K, Stahlhut C, Orellana E, Zhao J, Shimer E, Dysart S, Chen X, Bader AG and Slack FJ: A combinatorial microRNA therapeutics approach to suppressing non-small cell lung cancer. Oncogene 34: 3547-3555, 2015.

2. Fassina A, Cappellesso R and Fassan M: Classification of non-small cell lung carcinoma in transthoracic needle specimens using microRNA expression profiling. Chest 140: 1305-1311, 2011.

3. Reungwetwattana T, Weroha SJ and Molina JR: Oncogenic pathways, molecularly targeted therapies, and highlighted clinical trials in non-small-cell lung cancer (NSCLC). Clin Lung Cancer 13: 252-266, 2012.

4. Horvath S, Zhang B, Carlson M, Lu KV, Zhu S, Felciano RM, Laurance MF, Zhao W, Qi S, Chen Z, et al: Analysis of oncogenic signaling networks in glioblastoma identifies ASPM as a molecular target. Proc Natl Acad Sci USA 103: 17402-17407, 2006. 
5. Hutter $\mathrm{C}$ and Zenklusen JC: The cancer genome atlas: Creating lasting value beyond its data. Cell 173: 283-285, 2018.

6. Yang C, Sun C, Liang X, Xie S, Huang J and Li D: Integrative analysis of microRNA and mRNA expression profiles in non-small-cell lung cancer. Cancer Gene Ther 23: 90-97, 2016.

7. Friedman RC, Farh KK, Burge CB and Bartel DP: Most mammalian mRNAs are conserved targets of microRNAs. Genome Res 19: 92-105, 2009.

8. Reddy KB: MicroRNA (miRNA) in cancer. Cancer Cell Int 15 : $38,2015$.

9. Yanaihara N, Caplen N, Bowman E, Seike M, Kumamoto K, Yi M, Stephens RM, Okamoto A, Yokota J, Tanaka T, et al: Unique microRNA molecular profiles in lung cancer diagnosis and prognosis. Cancer Cell 9: 189-198, 2006

10. Lee HY, Han SS, Rhee H, Park JH, Lee JS, Oh YM, Choi SS, Shin SH and Kim WJ: Differential expression of microRNAs and their target genes in non-small-cell lung cancer. Mol Med Rep 11: 2034-2040, 2015.

11. Gallardo E, Navarro A, Viñolas N, Marrades RM, Diaz T, Gel B Quera A, Bandres E, Garcia-Foncillas J, Ramirez J and Monzo M miR-34a as a prognostic marker of relapse in surgically resected non-small-cell lung cancer. Carcinogenesis 30: 1903-1909, 2009

12. Wei JS, Song YK, Durinck S, Chen QR, Cheuk AT, Tsang P, Zhang Q, Thiele CJ, Slack A, Shohet J and Khan J: The MYCN oncogene is a direct target of miR-34a. Oncogene 27: 5204-5213, 2008.

13. Mitchell KA, Zingone A, Toulabi L, Boeckelman J and Ryan BM: Comparative transcriptome profiling reveals coding and noncoding RNA differences in NSCLC from African Americans and European Americans. Clin Cancer Res 23: 7412-7425, 2017.

14. Irizarry RA, Hobbs B, Collin F, Beazer-Barclay YD, Antonellis KJ, Scherf U and Speed TP: Exploration, normalization, and summaries of high density oligonucleotide array probe level data. Biostatistics 4: 249-264, 2003.

15. Dweep H and Gretz N: miRWalk2.0: A comprehensive atlas of microRNA-target interactions. Nat Methods 12: 697, 2015.

16. Hsu SD, Chu CH, Tsou AP, Chen SJ, Chen HC, Hsu PW, Wong YH, Chen YH, Chen GH and Huang HD: miRNAMap 2.0: Genomic maps of microRNAs in metazoan genomes. Nucleic Acids Res 36 (Database Issue): D165-D169, 2008.

17. Shannon P, Markiel A, Ozier O, Baliga NS, Wang JT, Ramage D, Amin N, Schwikowski B and Ideker T: Cytoscape: A software environment for integrated models of biomolecular interaction networks. Genome Res 13: 2498-2504, 2003.

18. Kanehisa M and Goto S: KEGG: Kyoto encyclopedia of genes and genomes. Nucleic Acids Res 28: 27-30, 2000.

19. Yu G, Wang LG, Han Y and He QY: clusterProfiler: An R package for comparing biological themes among gene clusters OMICS 16: 284-287, 2012

20. Ashburner M, Ball CA, Blake JA, Botstein D, Butler $\mathrm{H}$, Cherry JM, Davis AP, Dolinski K, Dwight SS, Eppig JT, et al: Gene ontology: Tool for the unification of biology: The Gene Ontology Consortium. Nat Genet 25: 25-29, 2000.

21. Huang da W, Sherman BT and Lempicki RA: Systematic and integrative analysis of large gene lists using DAVID bioinformatics resources. Nat Protoc 4: 44-57, 2009.

22. Huang da W, Sherman BT and Lempicki RA: Bioinformatics enrichment tools: Paths toward the comprehensive functional analysis of large gene lists. Nucleic Acids Res 37: 1-13, 2009.

23. Szklarczyk D, Franceschini A, Kuhn M, Simonovic M, Roth A, Minguez P, Doerks T, Stark M, Muller J, Bork P, et al: The STRING database in 2011: Functional interaction networks of proteins, globally integrated and scored. Nucleic Acids Res 39 (Database Issue): D561-D568, 2011

24. Bandettini WP, Kellman P, Mancini C, Booker OJ, Vasu S, Leung SW, Wilson JR, Shanbhag SM, Chen MY and Arai AE: MultiContrast delayed enhancement (MCODE) improves detection of subendocardial myocardial infarction by late gadolinium enhancement cardiovascular magnetic resonance: A clinical validation study. J Cardiovasc Magn Reson 14: 83, 2012.

25. Therneau T and Lumley T: Survival: Survival analysis, including penalised likelihood. R package version 2.36-5. Survival: Survival analysis, including penalised likelihood $\mathrm{R}$ package version 2.36-2.2010, 2011.

26. Zhang B, Kirov S and Snoddy J: WebGestalt: An integrated system for exploring gene sets in various biological contexts. Nucleic Acids Res 33: W741-W748, 2005.
27. Braybrooke JP, O'Byrne KJ, Propper DJ, Blann A, Saunders M, Dobbs N, Han C, Woodhull J, Mitchell K, Crew J, et al: A phase II study of razoxane, an antiangiogenic topoisomerase II inhibitor, in renal cell cancer with assessment of potential surrogate markers of angiogenesis. Clin Cancer Res 6: 4697-4704, 2000.

28. Terashima M, Ichikawa W, Ochiai A, Kitada K, Kurahashi I, Sakuramoto S, Katai H, Sano T, Imamura H and Sasako M; ACTS-GC Group: TOP2A, GGH, and PECAM1 are associated with hematogenous, lymph node, and peritoneal recurrence in stage II/III gastric cancer patients enrolled in the ACTS-GC study. Oncotarget 8: 57574-57582, 2017.

29. Kodiakov DS, Klimachëv VV, Avdalian AM, Bobrov IP, Lazarev AF, Lushnikova EL and Nepomiashchikh LM: Topoisomerase IIa expression in correlation with clinical and morphological parameters and proliferation (based on argyrophilic proteins of nucleolar organizer regions and $\mathrm{Ki}-67$ antigen) in lung adenocarcinoma. Vopr Onkol 60: 63-68, 2014 (In Russian).

30. Nakayama Y and Yamaguchi N: Role of cyclin B1 levels in DNA damage and DNA damage-induced senescence. Int Rev Cell Mol Biol 305: 303-337, 2013

31. Yuan J, Krämer A, Matthess Y, Yan R, Spänkuch B, Gätje R, Knecht R, Kaufmann M and Strebhardt K: Stable gene silencing of cyclin B1 in tumor cells increases susceptibility to taxol and leads to growth arrest in vivo. Oncogene 25: 1753-1762, 2006.

32. Soria JC, Jang SJ, Khuri FR, Hassan K, Liu D, Hong WK and Mao L: Overexpression of cyclin B1 in early-stage non-small cell lung cancer and its clinical implication. Cancer Res 60: 4000-4004, 2000

33. Vogelstein B, Lane D and Levine AJ: Surfing the p53 network. Nature 408: 307-310, 2000

34. Jung IL, Kang HJ, Kim KC and Kim IG: PTEN/pAkt/p53 signaling pathway correlates with the radioresponse of non-small cell lung cancer. Int J Mol Med 25: 517-523, 2010.

35. Altieri DC: The molecular basis and potential role of survivin in cancer diagnosis and therapy. Trends Mol Med 7: 542-547, 2001.

36. Li F: Survivin study: What is the next wave? J Cell Physiol 197: 8-29, 2003.

37. Shivapurkar N, Reddy J, Chaudhary PM and Gazdar AF: Apoptosis and lung cancer: A review. J Cell Biochem 88 885-898, 2003.

38. Zhao H, Zhang X, Han Z, Wang Z and Wang Y: Plasma anti-BIRC5 IgG may be a useful marker for evaluating the prognosis of nonsmall cell lung cancer. FEBS Open Bio 8: 829-835, 2018

39. Zhu H, Wu H, Liu X, Li B, Chen Y, Ren X, Liu CG and Yang JM: Regulation of autophagy by a beclin 1-targeted microRNA, miR-30a, in cancer cells. Autophagy 5: 816-823, 2009.

40. Zhu J, Zeng Y, Xu C, Qin H, Lei Z, Shen D, Liu Z and Huang JA Expression profile analysis of microRNAs and downregulated miR-486-5p and miR-30a-5p in non-small cell lung cancer. Oncol Rep 34: 1779-1786, 2015.

41. Voronkova M, Luanpitpong S, Riedel H and Rojanasakul Y: Abstract 1913: SOX9 regulates cancer stem-like cells in non-small cell lung cancer. Cancer Res 77: 1913, 2017.

42. Świtlik W, Karbownik MS, Suwalski M, Kozak J and Szemraj J: miR-30a-5p together with miR-210-3p as a promising biomarker for non-small cell lung cancer: A preliminary study. Cancer Biomark 21: 479-488, 2018.

43. Yu L, Todd NW, Xing L, Xie Y, Zhang H, Liu Z, Fang H, Zhang J, Katz RL and Jiang F: Early detection of lung adenocarcinoma in sputum by a panel of microRNA markers. Int J Cancer 127: 2870-2878, 2010

44. Yu JR, Tai Y, Jin Y, Hammell MC, Wilkinson JE, Roe JS, Vakoc CR and Van Aelst L: TGF- $/$ /Smad signaling through DOCK4 facilitates lung adenocarcinoma metastasis. Genes Dev 29: 250-261, 2015.

45. Zhan P, Xi GM, Zhang B, Wu Y, Liu HB, Liu YF, Xu WJ, Zhu Q, Cai F, Zhou ZJ, et al: NCAPG2 promotes tumour proliferation by regulating $\mathrm{G} 2 / \mathrm{M}$ phase and associates with poor prognosis in lung adenocarcinoma. J Cell Mol Med 21: 665-676, 2017.

46. Hu H, Li S, Liu J and Ni B: MicroRNA-193b modulates proliferation, migration, and invasion of non-small cell lung cancer cells. Acta Biochim Biophys Sin (Shanghai) 44: 424-430, 2012.

This work is licensed under a Creative Commons Attribution-NonCommercial-NoDerivatives 4.0 International (CC BY-NC-ND 4.0) License. 